\title{
En plena crisis económica: coste y efectividad de las unidades de estancia corta hospitalarias
}

\section{Economic crisis: cost and effectiveness of short stay hospital units}

\author{
F. Epelde, M.L. Iglesias-Lepine, L. Anarte
}

\section{RESUMEN}

Fundamento. La duración de la estancia es el principal determinante del coste de una hospitalización, por lo que se deben implementar estrategias para reducir la estancia hospitalaria convencional manteniéndose los niveles de calidad. Las Unidades de Estancia Corta (UEC) nacen con el objetivo de disminuir la estancia hospitalaria en un grupo de patologias y pacientes determinados. El objetivo de este original es evaluar la bibliografía existente sobre la evidencia de la disminución del periodo de estancia, eficiencia, reconsultas en los servicios de urgencias, coste-efectividad, y mortalidad de estas unidades.

Método. Se realizó una revisión sistemática de las publicaciones que aparecen en la literatura, utilizando los términos MeSH "Observation Unit", "Short-Stay Ward", "Monday to Friday Clinic", "Monaday to Friday Surgery Ward", "Short Stay Hospitalization", "Alternative to Convencional Hospitalization", "Alternative Admissions" en la base de datos MEDLINE, Web of Knowledge y la Cochrane Library desde el 1 de enero de 1960 al 1 de enero del 2012. Los estudios revisados se seleccionaron según la US Preventive Services Task Force Protocol.

Resultados. Las UEC permiten en grupos determinados de pacientes, disminuir el periodo de estancia, mantener la eficiencia, no mostrando incrementos de los reingresos por urgencias, ni la mortalidad.

Conclusiones. Las UEC pueden ser un instrumento para disminuir el coste del proceso sanitario en un grupo de patologias determinadas.

Palabras clave. Unidades de estancia corta. Alternativa a la hospitalización.

\begin{abstract}
Background. Length of stay is the main determinant of the cost of hospitalization, which is why strategies must be implemented to reduce conventional hospital stays while maintaining quality levels. Short Stay Units (SSU) were created with the aim of reducing hospital stays in a certain group of patients and pathologies. The aim of this paper is to evaluate the literature on the evidence of decreased length of stay, efficiency, readmissions in the emergency department, cost-effectiveness, and mortality of these units.
\end{abstract}

Methods. We made a systematic review of the literature, using the MeSH terms "Observation Unit", "ShortStay Ward", "Monday to Friday Clinic", "Monday to Friday Surgery Ward", "Short Stay Hospitalization", "Alternative to Conventional Hospitalization", "Alternative Admissions" in the MEDLINE database, Web of Knowledge and the Cochrane Library from January 1 1960 to January 12012 . The studies reviewed were selected according to the U.S. Preventive Services Task Force Protocol.

Results. The SSU made it possible in certain groups of patients to reduce the period of stay and maintain efficiency, showing no increases in emergency readmissions or mortality.

Conclusions. The SSU can be an instrument to reduce the cost of the health process in a certain group of pathologies.

Key words. Short stay unit. Alternative to hospitalization.
Servicio de Urgencias. Hospital de Sabadell. Corporació Sanitária Parc Taulí. Sabadell.

Institut Universitari Parc Taulí. UAB.

Recepción: 20 de febrero de 2012

Aceptación provisional: 20 de marzo de 2012

Aceptación definitiva: 20 de junio de 2012

\author{
Correspondencia: \\ Francisco Epelde \\ Servicio de Urgencias \\ Hospital de Sabadell \\ Parc Taulí, S/N \\ 0228 Sabadell \\ Barcelona \\ E-mail: fepelde@gmail.com
}




\section{INTRODUCCIÓN}

El coste de la sanidad española representó en el año 2009 el 9,5 \% del Producto Interior Bruto (PIB), con una previsión de incremento del $12 \%$ en el año $2012^{1}$. Los factores demográficos, el envejecimiento de la población, la tasa de cobertura o el gasto en las nuevas tecnologías y la evolución de las prácticas médicas (procesos más graves y complejos) son las características que más penalizan el gasto público sanitario ${ }^{2-3}$. Para intentar contener el progresivo incremento, en una época de crisis económica, la duración de la estancia es el principal determinante del coste de una hospitalización, por lo que se deben implementar estrategias para reducir la estancia hospitalaria convencional manteniéndose los niveles de calidad ${ }^{3}$. Las Unidades de Estancia corta (UCE) se están implantando como medida a las Unidades de Hospitalización Convencional (UHC) ${ }^{4-6}$.

Las UCE nacen con la hipótesis de que la eficiencia y calidad de la atención a un paciente que no requiere una estancia hospitalaria prolongada, puede mejorar si es ingresado en una unidad diferenciada ${ }^{7-8}$. Las UCE son unidades de hospitalización para patologias en las cuales los estudios a realizar y su estancia media se prevé limitada, generalmente hasta 96 horas $^{9-10}$. El tipo de patologías tratadas en nuestro país suelen ser de Medicina Interna General, aunque en la literatura existen referencias a UCE monográficas. La gran mayoría son pacientes que suelen ser dados de alta al domicilio. Normalmente las patologías a ingresar son médicas, lo que representa una actividad continuada de los médicos internistas, que incluye noches y festivos que las diferencia probablemente de las UHC. Por este último motivo, suelen estar adscritas a los servicios de urgencias, al facilitar la cobertura médica de 24 horas.

El objetivo de la revisión es analizar la literatura existente sobre las UEC y valorar si existe evidencia sobre su efectividad y su eficiencia en comparación con las UHC.

\section{MÉTODO}

Se realizó una revisión sistemática de las publicaciones que aparecen en la literatura, utilizando los términos MeSH "Observation Unit", "Short-Stay Ward", "Monday to Friday Clinic", "Monaday to Friday Surgery Ward", "Short Stay Hospitalization", "Alternative to Convencional Hospitalization", "Alternative Admissions" en la base de datos MEDLINE la Web of Knowledge y la Cochrane Library desde el 1 de enero de 1960 al 1 de enero del 2012.

Los estudios revisados se seleccionaron según la US Preventive Services Task Force Protocol ${ }^{11}$, catalogándolos en los siguientes apartados: I: Estudios con evidencia obtenida al menos por un estudio aleatorizado. II-1: Evidencia obtenida por estudios controlados no aleatorizados. II-2: Evidencia obtenida de estudios de cohortes o estudios de caso-control. II-3: Evidencia obtenida de estudios de series. III: Evidencia obtenida de estudios descriptivos. IV: Opiniones de autoridades, consensos o experiencia clínica. Se desecharon para el análisis los estudios basados en opiniones de expertos.

\section{RESULTADOS}

Los resultados a la revisión bibliográfica se exponen en las tablas 1 y 2 .

En la tabla 1 se recoge el nivel de evidencia de los estudios publicados. La mayoría de los estudios son comparativos ${ }^{12}$. Cinco de ellos son aleatorizados, pero el impacto de la UEC puede ser confundido por la implantación de un protocolo para el tratamiento de una patología específica que no fue utilizado en el grupo control, por lo que no queda claro si los resultados observados son atribuibles exclusivamente a la UEC.

En la tabla 2 se resume la evidencia observada con respecto a la calidad de atención, el tiempo de estancia, la eficiencia, las reconsultas en el SUH, los ingresos hospitalarios, el coste-efectividad, la satisfacción del paciente y la mortalidad. 
Tabla 1. Nivel de evidencia de los estudios publicados

\begin{tabular}{|c|c|c|}
\hline Estudios & Número de pacientes & Nivel de evidencia \\
\hline Willert y col, $1985^{18}$ & 103 & I \\
\hline Gomez y col, $1996^{14}$ & 100 & I \\
\hline McDermott y col, $1997^{19}$ & 222 & I \\
\hline Farkouh y col, $1998^{8}$ & 424 & I \\
\hline Rydman y col, $1998^{13}$ & 113 & I \\
\hline MacLaren y col, $1993^{31}$ & 405 & II-1 \\
\hline Gaspoz y col, $1994^{30}$ & 1.453 & II-1 \\
\hline Brillman y col, $1994^{20}$ & 1.224 & II-1 \\
\hline Bazarian y col, $1996^{16}$ & 1.424 & II-1 \\
\hline Hadden y col, $1996^{29}$ & 214 & II-1 \\
\hline Gouin y col, $1997^{17}$ & 4.227 & II-1 \\
\hline Saunders y col, $1988^{14}$ & 54 & II-2 \\
\hline Broquetas y col, $2008^{23}$ & 294 & $\mathrm{II}-2$ \\
\hline Puig y col, $2007^{24}$ & 2.915 & $\mathrm{II}-2$ \\
\hline Juan y col, $2006^{28}$ & 5.666 & III \\
\hline Broquetas y col, $2008^{27}$ & 147 & III \\
\hline Juan y col, $2011^{26}$ & 606 & III \\
\hline
\end{tabular}

Tabla 2. Relación de la bibliografía respecto a los parámetros estudiados

\begin{tabular}{lrrr}
\hline & Mejora & No se modifica & Disminuye \\
\hline Situación clínica & - & $8-13$ & - \\
\hline Periodo de estancia & $26-28$ & 15 & 14 \\
\hline Eficiencia & 16 & - & - \\
\hline Reconsultas en el SUH & 17 & $18-24$ & 23 \\
\hline Ingresos ordinarios & - & $8-20-26-27$ & $17-19$ \\
\hline Coste efectividad & - & - & - \\
\hline Calidad de vida del paciente & 13 & - & - \\
\hline Satisfacción del paciente & $15-21-28$ & $23-24$ & - \\
\hline Mortalidad & - & & 13 \\
\hline
\end{tabular}

Calidad de la atención. Dos estudios randomizados evidenciaron una equivalencia en las altas de los pacientes en el tratamiento habitual recibido para el dolor torácico y el asma ${ }^{8,13}$. Farkouh y col $^{8}$ compararon los resultados de 6 meses en 424 pacientes con ángor de riesgo intermedio (inclusión: infarto agudo de miocardio, insuficiencia cardíaca, ictus y paro cardiaco prehospitalario), que fueron aleatorizados para recibir tratamiento en una unidad de dolor torácico o en una $\mathrm{UHC}^{8}$. Se apreciaron 18 eventos en el grupo que fue asignado a la UHC y 14 en el grupo ingresado en 
la UEC. La diferencia entre los dos grupos no fue estadísticamente significativa. Tampoco se apreciaron diferencias entre ambos grupos en los reingresos en el SUH por dolor torácico, revascularización cardiaca, utilización de exploraciones complementarias y hospitalización por problemas cardiacos. Rydman y col ${ }^{13}$ evaluaron a 113 pacientes con exacerbación aguda del asma que no mejoró tras 3 horas de estancia en el SUH. Los pacientes se aleatorizaron en dos grupos, un grupo se ingresó en la UEC y el otro en la UHC. No se apreciaron diferencias significativas en la evolución de los pacientes medida por "peak flow" ni en la supervivencia a las 8 semanas. Ningún estudio con nivel de evidencia I o II ha mostrado una superioridad ni inferioridad en los pacientes que han sido ingresados en UCE.

Tiempo de estancia. En el estudio aleatorizado y controlado publicado por Gómez y col $^{14}$ que incluyó a 100 pacientes con dolor torácico, evaluaron la eficacia de un protocolo de diagnóstico rápido en pacientes con dolor torácico. La estancia media de los pacientes que fueron ingresados con la UEC fue de 15,4 $\pm 12,2 \mathrm{~h}$ frente a las $54,6 \pm 12,6 \mathrm{~h}$ en los pacientes que recibieron la atención en la UHC. Dado que la mejora de la estancia fue atribuida por los autores a la implantación de un nuevo protocolo de diagnóstico, es difícil considerar el papel que desempeñó la UEC. Saunders y col $^{15}$ examinaron las exacerbaciones de la pancreatitis crónica en 27 pacientes consecutivos que fueron aleatorizados para ingresar en la UEC o en la UHC. Catorce de los pacientes ingresados en la UEC fueron dados de alta en las primeras 24 horas con una estancia media de 14,4 horas. Los 13 pacientes que fueron ingresados en la UHC tuvieron una media de 7,5 días aunque los pacientes ingresados en la UEC tenían un valor de amilasas menor que los ingresados en la UHC.

Eficiencia. Bazarian y col ${ }^{16}$ examinaron el impacto de una UEC en la disminución de los ingresos hospitalarios. La media de los pacientes que permanecieron en el SUH más de 8 horas en espera de una cama en la planta cuando se implantó la UEC pasó de 9,6 \pm 4,2 h (antes de la implantación de la UEC) a 2,3 $\pm 2,6 \mathrm{~h}$ tras la implantación de la misma. Los autores confirman que tras la el inicio de la UEC disminuyó el tiempo de tratamiento de los pacientes de 7,3 a 5,5 horas en los pacientes con dolor torácico y de 5 a 4,2 horas en los pacientes con asma, existiendo diferencias estadísticamente significativas. Estos cambios podrían, no obstante, ser debidos al incremento de camas hospitalarias de 722 a 736 en el periodo de tiempo estudiado. Por lo que no existen estudios de nivel I o II que indiquen superioridad o inferioridad de las UEC frente a las UHC.

Reconsultas en el SUH. Existen dos trabajos que valoran el impacto de la UEC en los reingresos de los pacientes con asma en el SUH. Gouin y col $^{17}$ evidenciaron que existía un incremento de reconsultas en las primeras $72 \mathrm{~h}$ (del 3 al 5\%) al iniciar la actividad de la UEC. El estudio randomizado de Willert y col $^{18}$ con 103 niños con asma evidenció que no existían diferencias entre los grupos tratados en la UEC o en la UHC.

Ingresos hospitalarios. Evaluando el impacto de la UEC en el número de pacientes que ingresan por dolor torácico, Farkouh $\mathrm{y} \mathrm{col}^{8}$ en su estudio incluyó 212 pacientes con dolor torácico de riesgo intermedio, apreciándose una disminución de ingresos en la UHC al ser ingresados en una UEC. Mc Dermott y col $^{19}$ en su estudio aleatorizado controlado en pacientes con asma que no pudieron ser dados de alta tras 3 horas de estancia en el SUH apreciaron que en el grupo de 110 pacientes tratados en una UEC, el 59\% de los pacientes fueron dados de alta a domicilio y el $41 \%$ fue ingresado en la UEC. Brillman y col $^{20}$ estudiaron la evolución de 834 pacientes antes de abrir una UHC y 390 pacientes tras abrir la UEC. Evaluaron que el uso de una UEC en pacientes con asma no redujo la hospitalización por dicha patología.

Coste-efectividad. Rydman y $\operatorname{col}^{13}$ evaluaron los costes de los pacientes atendidos en una UEC versus una UHC por asma. El coste total por paciente fue obtenido sumando todos las asistencias recibidas por el paciente. La conclusión de los autores fue que la UCE tenía un coste significativa- 
mente menor $(1.203 \pm 1.344$ dólares USA) que los ingresados en la UHC $(2.247 \pm 110$ dólares USA) ${ }^{16}$. El coste medio de los pacientes atendidos en la UEC y que posteriormente precisaron hospitalización convencional al no mejorar su patología fue discretamente superior $(2.270 \pm 967$ dólares USA), lo que posiblemente se traduzca por una mayor complejidad ${ }^{12}$.

Satisfacción del paciente. Ha sido valorada en estudios aleatorizaros dos tipos de patología: el dolor torácico y el asma. Rydman y col ${ }^{21}$ evaluaron los niveles de satisfacción en pacientes con dolor torácico que ingresaron en la UEC. Un total de 104 pacientes fueron randomizados entre recibir tratamiento en la UEC o en la UHC. La satisfacción del paciente se analizó en una encuesta al alta. La conclusión de los autores fue que los pacientes ingresados en la UEC presentaron un nivel de satisfacción mayor que los ingresados en la UHC. Este estudio incorporó el uso de un protocolo de diagnostico rápido y por este motivo no se puede concluir que la mejoría de la satisfacción del los pacientes ingresados en la UEC sea debida a este motivo. Otro estudio de Rydman y col $^{22}$ valoró el nivel de satisfacción en 163 pacientes con asma con criterio de ingreso hospitalario tras 3 horas de tratamiento convencional en el SUH. Ochenta y un pacientes fueron aleatorizados e ingresados en la UEC y 82 en la UHC. Los pacientes en la UEC tenían escalas de satisfacción superior en referencia al trato recibido, la comunicación, el soporte emocional y el confort que pueden ser debidos a multitud de factores.

Mortalidad. Broquetas y $\mathrm{col}^{23}$ en un estudio aleatorizado de 294 pacientes demuestran que una UEC específica para pacientes neumológicos no incrementa la mortalidad frente a la UHC. Puig y $\operatorname{col}^{24}$ en una muestra de 2.915 pacientes aprecian que la mortalidad en pacientes diabéticos es similar en los pacientes atendidos en la UEC como en la UHC.

\section{DISCUSIÓN}

La revisión de la literatura consultada permite concluir que las UEC pueden ofrecer al paciente igual atención que en las UHC, reduciendo el periodo de estancia y por tanto los costes finales del proceso. Esta disminución del periodo de estancia no está necesariamente ligada al incremento de los reingresos, disminución de la calidad asistencial ni aumento de la mortalidad. Las UEC permiten disminuir los ingresos no necesarios en camas de hospitalización convencional, mejorar la atención percibida por el paciente y disminuir probablemente el porcentaje de infección nosocomial. Para el correcto funcionamiento de estas unidades se deben cumplir una serie de requerimientos ${ }^{14-25}$ : idealmente deben de encontrarse bajo manejo por médicos internistas; el tiempo de estancia debe limitarse a una máxima de 96 horas; los objetivos por paciente deben de estar claramente definidos; no deben de utilizarse como comodín cuando faltan camas en las UHC y si el paciente tuviera que ingresar, el ingreso debe tener prioridad sobre otros pacientes para poder mantener el índice de rotación pactado, lo que hace que las UEC sean unidades altamente dinámicas $^{26-28}$. En definitiva, podemos afirmar que en la gestión sanitaria, las UEC son una herramienta de optimización de los recursos sanitarios públicos ${ }^{29}$.

No obstante, este trabajo presenta limitaciones metodológicas ya que existe un número pequeño de publicaciones sobre el tema y existe poca evidencia de publicaciones de niveles I y II. La mayoría de los estudios tienen el factor de confusión de los resultados que publican por la instauración de un nuevo protocolo o circuito de tratamiento. Por otro lado, la diversidad de funcionamiento, estructura y dependencia jerárquica hace que sean difícilmente comparables entre $\mathrm{si}^{30}$. Las UEC españolas, de las que hay poca literatura publicada, suelen ser unidades médicas, de funcionamiento dispar y con dependencia jerárquica bien de servicios de urgencias o de Medicina Interna ${ }^{29-31}$. Estas unidades en ocasiones son difíciles de comparar con las UEC extranjeras con funcionamiento más consolidado y autónomo así como con un gran componente de especialización $^{32}$. 


\section{BIBLIOGRAFÍA}

1. Base de datos de la OCDE sobre la salud 2011. (Consultado el 4 de abril del 2012). Disponible en http://www.oecd.org/dataoecd/26/24/48406859.pdf.

2. Hammond CL, Pinnington LL, Phillips MF. A qualitative examination of inappropriate hospital admissions and lengths of stay. BMC Health Serv Res 2009; 5: 9-44.

3. Andreu-Ballester JC, Almela-Quilis A, Ballester F, Colomer-Rubio E, Marín-Tena E, Peiró A. Social and health characteristics of elderly patients with chronic and/or terminal diseases (PALET profile) attended in a short stay medical unit. Revista Espanola de Geriatria y Gerontologia 2006; 41: 327-333.

4. Hammond CL, Phillips MF, Pinnington LL, PearSON BJ, FAKIS A. Appropriateness of acute admissions and last in-patient day for patients with long term neurological conditions. BMC Health Serv Res 2009; 27: 9-40.

5. Moreno E, Garcia JM, Lea MC. Diferencias de gestión entre los ingresos urgentes y los programados en función de los grupos relacionados de diagnóstico y la edad de los pacientes. Emergencias 2007; 19: 122-128.

6. Juan A, Enjamio E, Moya C, García C, Castellanos J, PÉrez J et al. Impacto de la implementación de medidas de gestión hospitalaria para aumentar la eficiencia en la gestión de camas y disminuir la saturación del servicio de urgencias. Emergencias 2010; 22: 249-253.

7. SÁnchez M, Salgado E, Miró O. Mecanismos organizativos de adaptación y supervivéncia de los servicios de urgencias. Emergencias 2008; 20: 48-53.

8. Farkouh M, Smars P, Reeder G, Zinsmeister AR, Evans RW, Meloy TD et al. A clinical trial of a chest-pain observation unit for patients with unstable angina. N Engl J Med 1998; 339: 1882-1888.

9. Alonso G, Escudero JM. The emergency department short stay unit and the hospital at home as alternatives to standard inpatient hospitalisation. An Sist Sanit Navar 2010; 33 (Suppl 1): 97-106.

10. SÁnchez M, Santiago I. Specific organisational areas and preferential circuits for prevalent pathologies in emergency care. An Sist Sanit Navar 2010; 33 (Suppl 1): 89-96.

11. Mason J, Freemantle N, Nazareth I, Eccles M, Haines A, Drummond M. When is it cost-effective to change the behavior of health professionals? JAMA 2001; 19: 2988-2992.
12. The selection of acute medical admissions for a short-stay unit. Intern Emerg Med 2011; 6: 321-327.

13. Rydman RJ, Isola ML, Roberts RR, ZaLENSKI RJ, McDermott MF, Murphy DG et al. Emergency department observation unit versus hospital inpatient care for a chronic asthmatic population. Med Care 1998; 3: 599-609.

14. Gomez M, Anderson J, Karagounis L. An emergency department based protocol for rapidly ruling out myocardial ischaemia reduces hospital time and expense: results of a randomized study (ROMIO). J Am Coll Cardiol 1996; 28: 25-33.

15. Saunders C, Gentile D. Treatment of mild exacerbations of recurrent alcoholic pancreatitis in an emergency department observation unit. South Med J 1988; 81: 317-320.

16. Bazarian J, Schneider S, Newman V, Chodosh J. Do admitted patients held in the emergency department impact the throughput of treat and release patients? Acad Emerg Med 1996; 3: 1113-1118.

17. Gouin S, Macarthur C, Parkin P, Schuh S. Effect of a pediatric observation unit on the rate of hospitalization for asthma. Ann Emerg Med 1997; 29: 218-222.

18. Willert C, Davis AT, Herman JJ, Holson BB, ZieserL E. Short-term holding room treatment of asthmatic children. J Pediatr 1985; 106: 707-711.

19. McDermott MF, MuRPHy DG, Zalenski RJ, RYDman RJ, McCarRen M, Marder D et al. A comparison between emergency diagnostic and treatment unit and inpatient care in the management of acute asthma. Arch Intern Med 1997; 157: 2055-2062.

20. Brillman J, Tandberg D. Observation unit impact on emergency department admissions for asthma. Am J Emerg Med 1994; 12: 11-14.

21. Rydman RJ, Zalenski RJ, Roberts RR, Albrecht GA, Misiewicz VM, Kampe LM et al. Patient satisfaction with an emergency department chest pain observation unit. Ann Emerg Med 1997; 29: 109-115.

22. Rydman RJ, Roberts RR, Albrecht GL, ZaLEnski RJ, Mc DеRмотт M. Patient satisfaction with an emergency department asthma observation unit. Acad Emerg Med 1999; 6: 178-183.

23. Broquetas JM, Pedreny R, Martinez-Llorens J, SEllarésa J, Gea J. Short-stay respiratory unit: A new option for inpatient care. Archivos de Bronconeumologia 2008; 44: 252-256.

24. Puig J, Supervía A, Márquez M, Flores J, Cano JF, GutiérRez J. Diabetes team consultation: Impact on length of stay of diabetic patients admitted to a short-stay unit. Diabetes Res Clin Pract 2007; 78: 211-216. 
25. Abenhaim HA, Kahn SR, RafFoul J, Becker MR. Program description: A hospitalist-run, medical short-stay unit in a teaching hospital. CMAJ 2000; 16: 1477-1480.

26. Juan A, Jacob Rodríguez J, LLopis F, Gómez C. Análisis de la seguridad y la eficiencia de una Unidad de corta estancia en el tratamiento de la neumonia adquirida de la comunidad. Emergencias 2011; 3: 175-182.

27. Broquetas JM, Pedreny R, Martínez-Llorens JM, Sellarésa J, Gea J. Unidad neumológica de estancia corta: un nuevo dispositivo asistencial de hospitalización. Arch Bronconeumol 2008; 44: 252-256.

28. Juan A, Salazar A, Alvarez A, Pérez JR, Garcia L, CoRbella X. Effectiveness and safety of an Emergency department short-stay unit as an alternative to standard in patient hospitalization. Emerg Med J 2006; 23: 833-837.

29. Hadden D, Dearden C, Rocke L. Short stay observation patients: general wards are inappropriate. J Accid Emerg Med 1996; 13: 163165.

30. Gaspoz JM, LeE TH, Weinstein MC, Cook EF, Goldman P, Komaroff AL et al. Cost-effectiveness of a new short-stay unit to "rule out" acute myocardial infarction in low risk patients. J Am Coll Cardiol 1994; 24: 1249-1259.

31. Maclaren R, Ghoorahoo H, Kirby N. Use of an accident and emergency department observation ward in the management of head injury. Br J Surg 1993; 80: 215-217.

32. KROME R. Observation care units. Ann Emerg Med 1989; 18: 705. 